\title{
A REMARK ON FROBENIUS EXTENSIONS AND ENDOMORPHISM RINGS
}

\author{
TADASI NAKAYAMA and TOSIRO TSUZUKU
}

In his paper [1] F. Kasch developed a theory of Frobenius extensions as a generalization of the theory of Frobenius algebras. In it he established a very interesting relationship between the Frobenius property of an extension and that of its endomorphism ring [1, Satz 5], from which he further derived the Frobenius extension property of Galois extensions of simple rings [1, Satz 6]; with these results he kindly responded to what had been vaguely "conjectured" (as he wrote) by one of the writers on the connection between Galois theory and the theory of Frobenius algebras [2]. However, his theory of Frobenius extensions is constructed upon the assumption that the ground ring, $A$, satisfies the minimum condition (for left ideals and right ideals) and, moreover, the condition that the left annihilator, in $A$, of a right ideal in $A$ different from $A$ should not vanish and similarly the right annihilator, in $A$, of a left ideal different from $A$ should not vanish; he calls such a ring an S-ring. His proof to his above alluded theorem on the relationship between Frobenius extensions and endomorphism rings depends also to this assumption, and particularly to the last condition. The purpose of the present note is to tree the theorem from this condition (and even from the minimum condition) establishing it for the case of an arbitrary ground ring.

(It is desireable to free also some other parts of the theory from the same S-ring assumption, though for some parts of the theory the assumption is rather natural and perhaps necessary, and the job will be taken up in a subsequent paper, to appear somewhere, in which generalizations of the theory in other contexts will be considered too.)

\section{Preliminaries}

Let $\Xi$ be a ring having a unit element 1 , and $A$ be a subring of $\Xi$ which contains 1. The module $\operatorname{Hom}_{A}\left(\Xi_{A}, A_{A}\right)$ of $A$-right-homomorphisms of $\Xi$ into $A$

Received January 28, 1959. 
has an $A$-๔-module strunture defined by

$$
\begin{aligned}
& (a \varphi)(x)=a \varphi(x), \quad(\varphi s)(x)=\varphi(s x) \\
& \left(\varphi \in \operatorname{Hom}_{A}\left(\bigodot_{A}, A_{A}\right) ; a \in A ; x, s \in \Im\right) .
\end{aligned}
$$

We define $\subseteq$ to be a Frobenius extension of $A$ when $\subseteq$ has a finite independent right-basis over $A$ and when moreover there is an $A$ - $\subseteq$-isomorphism between the $A$ - $\Subset$-modules $\Subset$ and $\operatorname{Hom}_{A}\left(\bigodot_{A}, A_{A}\right)$.

If $\subseteq$ is a Frobenius extension of $A$ and if $\Phi$ is an $A$ - $\subseteq$-isomorphism of $\subseteq$ and $\operatorname{Hom}_{A}\left(\Theta_{A}, A_{A}\right)$, there exists for every finite independent $A$-right-basis ( $t_{1}$, $\left.\ldots, t_{n}\right)$ of $\Subset$ a finite independent $A$-left-basis $\left(s_{1}, \ldots, s_{n}\right)$ of $\Subset$ such that

$$
\emptyset\left(s_{\nu}\right)\left(t_{\mu}\right)=\emptyset(1)\left(s_{\nu} t_{\mu}\right)=\delta_{\mu \nu} 1 \text {. }
$$

Indeed, if $\varphi_{\nu}$ denotes, for each $\nu$, the $A$-right-homomorphism of $\subseteq$ into $A$ with $\varphi_{\nu}\left(t_{\mu}\right)=\delta_{\mu \nu} 1$, then we have readily the direct decomposition

$$
\operatorname{Hom}_{A}\left(\Im_{A}, A_{A}\right)=A \varphi_{1}+\cdots+A \varphi_{n},
$$

and we have simply to put $s_{\downarrow}=\Phi^{-1}\left(\varphi_{\downarrow}\right)$, in order to obtain our assertion, turning to $\Subset$ from $\operatorname{Hom}_{A}\left(\Subset_{A}, A_{A}\right)$.

If here

$$
x t_{\nu}=\sum_{\mu=1}^{n} t_{\mu} y_{\mu \nu}\left(y_{\mu \nu} \in A\right)
$$

for an element $x$ of $\Subset$, then we have

$$
s_{\mu} x=\sum_{\nu=1}^{n} y_{\mu \nu} s_{\nu}\left(y_{\mu \nu} \in A\right)
$$

with same $y_{\mu \nu}$. For, we have

$$
\emptyset(1)\left(s_{\mu} x t_{\nu}\right)=\emptyset(1)\left(s_{\mu} \sum_{\kappa} t_{\kappa} y_{\kappa \nu}\right)=y_{\mu \nu}
$$

if (1) is the case, while we should have

$$
\Phi(1)\left(s_{\perp} x t_{\nu}\right)=\emptyset(1)\left(\left(\sum_{\nu} z_{\mu \kappa} s_{\kappa}\right) t_{\nu}\right)=z_{\mu \nu}
$$

if $s_{\mu} x=\sum_{\kappa=1}^{n} z_{\mu \kappa} s_{\kappa}$.

Naturally (2) entails (1) too.

Conversely, if $\Theta$ has a finite independent $A$-right-basis $\left(t_{1}, \ldots, t_{n}\right)$ and a finite independent $A$-left-basis $\left(s_{1}, \ldots, s_{n}\right)$ such that (1), (2) entail each other, then $\Subset$ is a Frobenius extension of $A$. For, associating $s_{\downarrow}$ with the element $\psi_{\downarrow}$ 
of $\operatorname{Hom}_{A}\left(\Im_{A}, A_{A}\right)$ such that $\psi_{\nu}\left(t_{\mu}\right)=\delta_{\mu \nu} 1$, we obtain then an $A$ - $\Im$-isomorphism of $\Subset$ and $\operatorname{Hom}_{A}\left(\dot{\Im}_{A}, A_{A}\right)$.

Hence, $\Im$ is a Frobenius extension of $A$, if and only if $\Subset$ has a finite independent $A$-right-basis $\left(t_{1}, \ldots, t_{n}\right)$ and a finite independent $A$-left-basis $\left(s_{1}\right.$, $\ldots, s_{n}$ ) such that each of the relations (1), (2) entails the other.

This shows in particular that the notion of a Frobenius extension is rightleft symmetric and may be defined also by the existence of a finite independent $A$-left-basis of $\subseteq$ and an $\subseteq$ - $A$-isomorphism of $\subseteq$ and the module $\operatorname{Hom}_{A}\left({ }_{A} \cong,{ }_{A} A\right)$ of $A$-left-homomorphisms of $\subseteq$ into $A$.

Further, with a Frobenius extension $\Xi$ of $A$ and an $A$ - $\Xi$-isomorphism $\Phi$ of $\Im$ and $\operatorname{Hom}_{A}\left(\Im_{A}, A_{A}\right)$, consider the element $A=\emptyset(1)$ of $\operatorname{Hom}_{A}\left(\Im_{A}, A_{A}\right)$. Then $A$ is an $A$-two-sided homomorphism of $\Subset$ into $A$ and satisfies the conditions:

$\left.\mathrm{i}_{r}\right) \Lambda(s \Subset)=0(s \in \Im) \quad$ entails $s=0$,

$\left.\mathrm{i}_{l}\right) A(\Im s)=0(s \in \Im) \quad$ entails $s=0$,

ii) for every $\varphi$ in $\operatorname{Hom}_{A}\left(\Im_{A}, A_{A}\right)$ there exists an element $s$ in $\Xi$ with $\varphi(x)$ $=A(s x)$ (that is to say $\left.\operatorname{Hom}_{A}\left(\mathbb{S}_{A}, A_{A}\right)=1 \Xi\right)$.

Indeed, $A(s \Xi)=0$ means $\Phi(1)(s \Xi)=0, \emptyset(s)(\Xi)=0, \emptyset(s)=0$ which implies $s=0$. Further, $\Lambda\left(\Xi_{s}\right)=0$ means $\Phi(1)(\Xi s)=0, \Phi(\Xi)(s)=0, \operatorname{Hom}_{A}\left(\Xi_{A}, A_{A}\right)(s)=0$ which implies $s=0$ as we readily see from the $A$-right vector space property of $\Xi$. ii) is clear from $\Xi=1 \subseteq$ and that $\Phi$ is an $\Xi$-right isomorphism of $\Xi$ and $\operatorname{Hom}_{A}\left(\Xi_{A}, A_{A}\right)$. (We may further prove that we have $\operatorname{Hom}_{A}\left({ }_{A} \Xi{ }_{A} A\right)=\Xi A$, symmetrically to ii). But we do not use this in the present not.)

Conversely, if there is an $A$-two-sided homomorphism $A$ of $\Xi$ into $A$ satisfying $\left.\dot{\mathrm{i}}_{r}\right)$, ii), then we obtain an $A$ - $₫$-homomorphism $\Phi$ of $\subseteq$ into $\operatorname{Hom}_{A}\left(\Xi_{A}, A_{A}\right)$

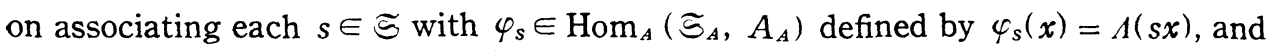
$\Phi$ is (monomorphic and epimorphic, whence) isomorphic (because of $i_{r}$ ), ii)).

Thus, under the assumption that $\subseteq$ has a finite independent right-basis over $A$, the existence of an $A$-two-sided homomorphism $A$ of $\subseteq$ into $A$ satisfying $\mathrm{i}_{r}$ ), $\mathrm{i}_{l}$ ) and $\left.\mathrm{ii}\right)$ is necessary and sufficient for $\Xi$ to be a Frobrnius extension of $A$.

Now, if we assume, as Kasch [1] did, that $A$ is an S-ring, ${ }^{11}$ then an $A$-twosided homomorphism $A$ of $\subseteq$ into $A$ satisfying $\mathrm{i}_{r}$ ), $\mathrm{i}_{l}$ ) satisfies ii) automatically, the existence of a finite independent $A$-right-basis of $\Xi$ being assumed again.

1) See the introduction. 
To see this, let $A$ be an $A$-two-sided homomorphism of $€$ into $A$ satisfying $\mathrm{i}_{r}$ ), $\dot{i}_{l}$ ). It defines a regular scalar product \langle\rangle in $A$ of the $A$-left-module $\Subset$ and the A-right-module $\subseteq$ by

$$
\langle x, z\rangle=\Lambda(x z) \quad(x, z \in \Subset) .
$$

Since $€$ has a finite independent right basis, say $\left(t_{1}, \ldots, t_{n}\right)$ and since $A$ is assumed to be an $S$-ring, there exists, by Satz $1^{2)}$ in Kasch [1], an $A$-leftsubmodule $\mathfrak{U}$ in $\Subset$ having an independent $A$-left-basis $\left(s_{1}, \ldots, s_{n}\right)$ orthogonal to $\left(t_{1}, \ldots, t_{n}\right)$ with respect to \langle\rangle$:\left\langle s_{\mu}, t_{\nu}\right\rangle=\delta_{\mu \nu} 1$. On the other hand, by associating $s \in \Subset$ with $\varphi_{s} \in \operatorname{Hom}_{A}\left(\Im_{A}, A_{A}\right)$ defined by $\varphi_{s}(x)=1(s x)$ we have an $A$-left (and $\Subset$-right) homomorphism $\Phi$ of $\Subset$ into $A$, which is monomorphic in virtue of $\left.\mathrm{i}_{r}\right)$. The $A$-left-module $\operatorname{Hom}_{A}\left(\Theta_{A}, A_{A}\right)$ has an independent $A$-(left-) basis $\left(\varphi_{1}, \ldots, \varphi_{n}\right)$ with $\varphi_{\mu}\left(t_{\nu}\right)=\delta_{\mu \nu} 1$. From $\operatorname{Hom}_{A}\left(\bigodot_{A}, A_{A}\right) \supseteq \emptyset(\Subset) \supseteq \Phi(\mathfrak{H})$ and a comparison of $A$-lengths we see $\operatorname{Hom}_{A}\left(\bigodot_{A}, A_{A}\right)=\emptyset(\Theta)$. This shows that ii) is the case too.

Thus, our definition of a Frobenius extension coincides with Kasch's in the case $A$ is an $\mathrm{S}$-ring (to which Kasch restricted his definition) at least a finite rank case (which his endomorphism ring theorem (Satz 5), as well as ours, deals with) is concerned. ${ }^{3)}$

\section{Theorem}

Let $\subseteq$ be a ring with unit element 1 , and $A$ a subring of $\subseteq$ containing 1 . The ring $\left(\xi\right.$ of (all) A-left-endomorphisms of $\Subset$ has a subring $\bigodot_{r}$ consisting of (all) the right multiplications of the elements of $\Subset$ onto $\Subset$ itself. $\Xi_{r}$ contains naturally the unit element of $\xi$, which is the identity map of $\mathbb{F}$.

We now prove the following refinement of Satz 5 in Kasch [1]:

Theorem. If $\Subset$ is a Frobenius extension of $A$, then the A-left-endomorphism ring $\left(5\right.$, of $\subseteq$ is a Frobenius extension of $\bigodot_{r}$. Conversely, if 5 is a Frobenius extension of $\Theta_{r}$, then $\Subset$ is a Frobenius extension of $A$, provided that $\Subset$ has a finite independent left-basis over A.

Proof. We begin with the second half of the theorem, and denote by

2) Observe that Kasch's [1] Satz 1 remains valid when the ambient modules $\mathscr{L}, \mathfrak{R}$ are not necessarily vector spaces, as an examination of his proof easily prevails.

3) We have seen moreover that in his definition the $A$-left-vecterspace property of $\subseteq$. is a consequence of the other parts of the definition, provided that $(\subseteq): A)_{r}$ is finite. 
$\left(s_{1}, \ldots, s_{n}\right)$ a finite independent $A$-left-basis of $\Xi$ :

$$
\Xi=A s_{1}+\cdots+A s_{n} .
$$

Let $E_{\nu}$ be the $A$-left-endomorphism of $\Xi$ such that

$$
s_{\mu}^{E_{\nu}}=\delta_{j \nu}, 1 .
$$

We readily see that $E_{1}, \ldots, E_{n}$ form an independent $\Xi_{r}$-right-basis of $[$ :

$$
\text { (F) }=E_{1} \Xi_{r}+\cdots+E_{n} \Xi_{r} .
$$

Now, by our assumption that $\left[5\right.$ is a Frobenius extension of $\Xi_{r}$, there exists an $\Xi_{r}$-two-sided homomorphism 1 of $\left(5\right.$ into $\Xi_{r}$ having the properties $\left.i_{r}\right)$, ii) in the preceding section, with $\Xi, A$ replaced by $\mathbb{E}$, $\Xi_{r}$. Set

$$
t_{\nu r}=\Lambda\left(E_{\nu}\right) \quad\left(t_{\nu} \in \Xi\right) ;
$$

with $x \in \Xi$ the right multiplication of $x$ onto $\Xi$ is denoted by $x_{r}$. We contend that a relation

$$
t_{1 r} y_{1 r}+\cdots+t_{n r} y_{n r}=0 \quad\left(y_{\nu} \in A\right)
$$

holds only when $y_{1}=\cdots=y_{n}=0$. To see this, put

$$
x_{i}=y_{\nu} s_{1}+\cdots+y_{\nu} s_{n}=y_{2}\left(s_{1}+\cdots+s_{n}\right) \in \Xi
$$

and consider an element

$$
X_{\mu}=\left(E_{1} x_{1 r}+\cdots+E_{n} x_{n r}\right) E_{i \iota}
$$

of $(5$. We have, for $a \in A$,

$$
\begin{aligned}
\left(a s_{\nu}\right)^{\tilde{x}_{\mu}} & =\left(a s_{\nu}\right)^{\left(E_{1} x_{x_{1}}+\cdots+E_{n} x_{n r}\right) E_{\mu}} \\
& =\left(a x_{\nu}\right)^{E_{\mu}}=\left(a y_{\nu}\left(s_{1}+\cdots s_{n}\right)\right)^{F_{\mu}}=a y_{\mu} .
\end{aligned}
$$

Since this is the case for each $\nu=1, \ldots, n$, we see

$$
X_{\mu}=E_{1} y_{1 r}+\cdots+E_{n} y_{n r} .
$$

Hence

$$
.1\left(X_{\mu}\right)=.1\left(E_{1} y_{1 r}+\cdots+E_{n} y_{n r}\right)=t_{1 r} y_{1 r}+\cdots+t_{n r} y_{n r}
$$

and this is 0 by the assumed relation (6). Then $.1\left(X_{\mu} x_{r}\right)=A\left(X_{\mu}\right) x_{r}=0$ for any $x \in \Xi$, i.e.

$$
.1\left(\left(E_{1} x_{1 r}+\cdots+E_{n} x_{n r}\right) E_{\mu} x_{r}\right)=0
$$

for any $x \in \Xi$. Since this is the case for $\mu=1, \ldots, n$, we have, in view of (5), 


$$
1\left(\left(E_{1} x_{1 r}+\cdots+E_{n} x_{n r}\right)(\xi)=0\right.
$$

and hence

$$
E_{1} x_{1 r}+\cdots+E_{n} x_{n r}=0
$$

by our cited property of $\Lambda$. We have then $x_{\nu}=0, y_{\nu}=0$ for $\nu=1, \ldots, n$, as was asserted.

So we obtain a direct sum submodule

$$
t_{1 r} A_{r}+\cdots+t_{n r} A_{r}
$$

of $\Theta_{r}$, where each $t_{v r} A_{r}$ is $A_{r}$-right-isomorphic to $A_{r} ; A_{r}$ denotes the totality of the right multiplications of the elements of $A$ onto $\subseteq$.

Next we prove that

$$
E_{1} A_{r}+\cdots+E_{n} A_{r}
$$

is a left ideal in $\xi=E_{1} \Xi_{r}+\cdots+E_{n} \Im_{r}$. Namely, with $x=y_{\mathrm{i}} s_{1}+\cdots+y_{n} s_{n} \in \Subset$ $\left(y_{2} \in A\right), y \in A$ we have

$$
\begin{aligned}
\left(a s_{\mu}\right)^{E_{\nu} x_{r} E_{\kappa} y_{r}} & =\left(\delta_{\mu \nu} a\right)^{x_{r} E_{\kappa} y_{r}} \\
& =\left(\delta_{\mu \nu} a\left(y_{1} s_{1}+\cdots+y_{n} s_{n}\right)\right)^{E_{\kappa} y_{r}}=\left(\delta_{\mu \nu} a y_{\kappa}\right)^{y_{r}} \\
& =\delta_{\mu \nu} a y_{\kappa} y
\end{aligned}
$$

for any $a \in A$ and $\kappa$, which means

$$
E_{\nu} x_{r} E_{\kappa} y_{r}=E_{\nu}\left(y_{\kappa} y\right)_{r} \in E_{\nu} A_{r} .
$$

This shows $\left(5 E_{\mathrm{\kappa}} A_{r} \subseteq E_{1} A_{r}+\cdots+E_{n} A_{r}\right.$ and, therefore, (7) is a left ideal in (5.

As $A$ is $\Xi_{r}$-left-(and $\Xi_{r}$-right-)homomorphic, it follows that $1\left(E_{1} A_{r}+\cdots\right.$ $\left.+E_{n} A_{r}\right)=t_{1 r} A_{r}+\cdots+t_{n r} A_{r}$ is a left ideal of $\Xi_{r}$ and indeed an $\Xi_{r}$-left, $A_{r}$ right-submodule of $\Xi_{r}$.

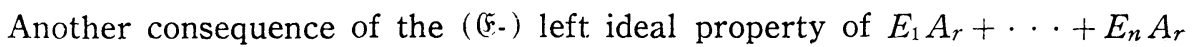
is, as we see by virtue of ii) (with $\Subset, A$ replaced by $\left(\xi, \varrho_{r}\right.$ ), that by any $\Xi_{r}$ right-homomorphism of (5. into $\Xi_{r}$ every element in $E_{1} A_{r}+\cdots+E_{n} A_{r}$ is mapped into $.1\left(E_{1} A_{r}+\cdots+E_{n} A_{r}\right)=t_{1 r} A_{r}+\cdots+t_{n r} A_{r}$. But, from (5) we see the

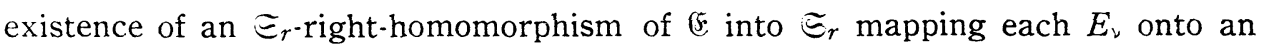
arbitrary element of $\Xi_{r}$. So we have $t_{1 r} A_{r}+\cdots+t_{n r} A_{r}(\supseteq$ whence $)=\Xi_{r}$. The left-hand side is a direct sum as we have seen. On turning to $\Xi$ we have a direct decomposition

$$
\Xi=t_{1} A+\cdots+t_{n} A
$$


of $\Xi$, where $t, A \approx A$ (A-right).

Further, if, with an element $x$ of $\Xi$, we have

$$
s_{\nu} x=\sum_{\mu} y_{\nu \mu} s_{\mu} \quad\left(y_{\nu \mu} \in A\right),
$$

then we have $x_{r} E_{\mu}=\sum_{\nu} E_{\nu} y_{\nu \mu r}$ as is seen from

$$
\begin{aligned}
\left(a s_{\nu}\right)^{x_{r} E_{\mu}} & =\left(a s_{\nu} x\right)^{E_{\mu}}=\left(a \sum_{\mu} y_{\nu \mu} s_{\mu}\right)^{E_{\mu}} \\
& =a y_{\nu \mu}=\left(a s_{\nu}\right) \sum_{\nu} E_{\nu} \nu_{\nu, l} \quad \quad(a \in A) .
\end{aligned}
$$

Applying $A$ we have $x_{r} t_{\mu r}=\sum_{\nu} t_{\nu r} y_{\nu \mu r}$, or

$$
x t_{\mu}=\sum_{\nu} t_{\nu} y_{\nu \mu}
$$

Hence $\subseteq$ is a Frobenius extension of $A$.

This proves the second half of our theorem. As to the first half, Kasch's proof holds good. For the sake of completeness we give here its modification adapted to our present treatment. Thus, assume that $\Xi$ is a Frobenius extension of $A$. We have (5) with (3), (4). Further we obtain the existence of an independent $A$-right-basis $\left(t_{1}, \ldots, t_{n}\right)$ of $\Xi$ such that (1), (2) (i.e. (10), (9)) entail each other. To each element $X$ of $\left(5\right.$ we associate an element $\varphi_{x}$ of Hom $\Xi_{r}\left(\Xi_{\Xi_{r}}\right.$, $\left.\left(\widetilde{\Xi}_{r}\right) \Xi_{r}\right)$ defined by

$$
\varphi_{X}(Y)=\sum_{i=1}^{r} t_{\nu r}\left(s_{i}^{X Y}\right)_{r} \quad(Y \in(\xi) .
$$

Then the map $X \rightarrow \varphi_{X}$ is an $\Xi_{r}$-left-homomorphism of $\left(\varepsilon\right.$ into Hom $\Xi_{r}\left(\varepsilon_{\Xi_{r}},\left(\Xi_{r}\right) \Xi_{r}\right)$, as we easily see from the relations (1), (2). The map is evidently (5-righthomomorphic too.

We wish to show that the map is an isomorphism. Let, for this purpose, $X=E_{1} x_{1 r}+\cdots+E_{n} x_{n r}\left(x_{\nu} \in \Xi\right)$ be a non-zero element of $(5$. There exists an index, say $\nu_{0}$, with $x_{\nu_{0}} \neq 0$. If we set $x_{\nu}=\sum y_{\nu \mu} s_{\mu}\left(y_{\nu \mu} \in A\right)$ for each $\nu$, then $y_{\nu_{0} \mu_{0}} \neq 0$ for some $\mu_{0}$. We have

$$
\begin{aligned}
\varphi_{X}\left(E_{\nu_{0}}\right) & =\sum_{\nu=0}^{n} t_{\nu r}\left(s_{\nu}^{\left.X F_{\mu_{0}}\right)_{r}}\right. \\
& =\sum_{\nu=0}^{n} t_{\nu r}\left(x_{\nu}^{F_{\mu 0}}\right)_{r}=\sum_{\nu=0}^{n} t_{\nu r}\left(y_{\nu \mu_{0}}\right)_{r} \\
& =\left(\sum_{\nu=1}^{n} t_{\nu} y_{\nu \mu_{0}}\right)_{r} \neq 0 .
\end{aligned}
$$

Hence $\varphi_{X} \neq 0$ and this proves that our map is monomorphic. 
Further, for arbitrarily given $a_{1}, \ldots, a_{n} \in A$, set

$$
X=\sum_{\nu=1}^{n} E_{\nu}\left(a_{\nu} s_{1}\right)_{r}
$$

Then

$$
\begin{aligned}
\varphi_{X}\left(E_{\mu}\right) & =\sum_{\nu=1}^{n} t_{\nu r}\left(s_{\nu}^{X E_{\mu}}\right)_{r}=\sum_{\nu=1}^{n} t_{\nu r}\left(\left(a_{\nu} s_{1}\right)^{E_{\mu}}\right)_{r} \\
& =\left\{\begin{array}{cc}
\sum_{\nu=1}^{n} t_{\nu r} a_{\nu r}=\left(\sum_{\nu=1}^{n} t_{\nu} a_{\nu}\right)_{r} & \text { for } \mu=1, \\
0 & \text { for } \mu \neq 1 .
\end{array}\right.
\end{aligned}
$$

Similarly, for each $\nu$ and for every element $x$ of $\subseteq$ there exists an $X$ in $\subseteq$ such that $\varphi_{X}\left(E_{\nu}\right)=x$ or 0 according as $\nu=\mu$ or $\nu \neq \mu$. This proves that our map is $X \rightarrow \varphi_{X}$ is epimorphic too.

Thus $\Xi_{r} E_{E} \approx \operatorname{Hom}_{\Xi_{r}}\left(E_{\Xi_{r}},\left(\Xi_{r}\right) \Xi_{r}\right)$ and $\mathbb{E}$ is a Frobenius extension or $\Xi_{r}$. The first half of our theorem is thus proved too.

Remark. In case $\subseteq$ satisfies the minimum condition for right ideals, the assumption of the existence of a finite independent $A$-left-basis of $\subseteq$ in the last part of our theorem may be weakened to the assumption of the existence of an independent $A$-left-basis or $\mathcal{S}_{\text {. }}$ For, if $\left(s_{1}, s_{2}, \ldots\right)$ is a such, then we obtain a direct sum submodule $E_{1} \Im_{r}+E_{2} \Im_{r}+\cdots$ of $[$. Since $[5$ is assumed to be a Frobenius extension of $\Theta_{r}$, this sum must be finite and our basis $\left(s_{1}, s_{2}, \ldots\right)$ must be finite too.

We remark further that even in case $A$ is an S-ring, (the second half of) our theorem provides a refinement of (the corresponding part of) Kasch's, since in the last the equality of the $A$-left and $A$-right ranks of $\cong$ is pre-assumed.

\section{REFERENCES}

[1] F. Kasch, Grundlagen einer Theorie der Frobeniuserweiterungen, Math. Ann. 127 (1954), 453-474.

[2] T. Nakayama, On two topics in the struntural theory of rings (Galois theory of rings and Frobenius algebras), Proc. Intern. Cngrews of Math. (1950), II, 49.

Mathematical Instatute

Nagoya University 